University of Nebraska - Lincoln

DigitalCommons@University of Nebraska - Lincoln

Publications, Agencies and Staff of the U.S.

Department of Commerce

U.S. Department of Commerce

$9-2008$

\title{
A General Framework for the Analysis of Animal Resource Selection from Telemetry Data
}

\author{
Devin S. Johnson \\ National Marine Mammal Laboratory, Alaska Fisheries Science Center, National Marine Fisheries Service \\ Dana L. Thomas \\ University of Alaska Fairbanks, Fairbanks \\ Jay M. Ver Hoef \\ National Marine Mammal Laboratory, Alaska Fisheries Science Center, National Marine Fisheries Service \\ Aaron Christ \\ Alaska Department of Fish and Game
}

Follow this and additional works at: https://digitalcommons.unl.edu/usdeptcommercepub

Part of the Environmental Sciences Commons

Johnson, Devin S.; Thomas, Dana L.; Ver Hoef, Jay M.; and Christ, Aaron, "A General Framework for the Analysis of Animal Resource Selection from Telemetry Data" (2008). Publications, Agencies and Staff of the U.S. Department of Commerce. 190.

https://digitalcommons.unl.edu/usdeptcommercepub/190

This Article is brought to you for free and open access by the U.S. Department of Commerce at DigitalCommons@University of Nebraska - Lincoln. It has been accepted for inclusion in Publications, Agencies and Staff of the U.S. Department of Commerce by an authorized administrator of DigitalCommons@University of Nebraska - Lincoln. 


\title{
A General Framework for the Analysis of Animal Resource Selection from Telemetry Data
}

\author{
Devin S. Johnson, ${ }^{1, *}$ Dana L. Thomas, ${ }^{2}$ Jay M. Ver Hoef, ${ }^{1}$ and Aaron Christ ${ }^{3}$ \\ ${ }^{1}$ National Marine Mammal Laboratory, Alaska Fisheries Science Center, National Marine \\ Fisheries Service, Seattle, Washington 98115, U.S.A. \\ ${ }^{2}$ Department of Mathematics and Statistics, University of Alaska Fairbanks, Fairbanks, Alaska 99709, U.S.A \\ ${ }^{3}$ Alaska Department of Fish and Game, Anchorage, Alaska 99518, U.S.A. \\ *email: devin.johnson@noaa.gov
}

\begin{abstract}
SUMmARY. We propose a general framework for the analysis of animal telemetry data through the use of weighted distributions. It is shown that several interpretations of resource selection functions arise when constructed from the ratio of a use and availability distribution. Through the proposed general framework, several popular resource selection models are shown to be special cases of the general model by making assumptions about animal movement and behavior. The weighted distribution framework is shown to be easily extended to readily account for telemetry data that are highly autocorrelated; as is typical with use of new technology such as global positioning systems animal relocations. An analysis of simulated data using several models constructed within the proposed framework is also presented to illustrate the possible gains from the flexible modeling framework. The proposed model is applied to a brown bear data set from southeast Alaska.
\end{abstract}

KEY WORDS: Autocorrelation; Brown bear; Discrete choice; Persistence; Resource selection; Telemetry.

\section{Introduction}

The ever-increasing availability of new technology to wildlife researchers has led to the frequent use of telemetry data for assessing habitat characteristics selected by animals. The advent of satellite technology, such as global positioning systems (GPS), has allowed wildlife researchers to collect telemetry data on a temporal scale that was not previously practical. Observations can now be obtained many times per day for many months with GPS devices. This development has inevitably led to a serious challenge to the usual independence assumption present in many animal resource selection studies (see Manly et al., 2002). Traditionally, data are removed from the sample until it is determined that an independence assumption is valid (Swihart and Slade, 1985). We believe that this removal of data defeats the purpose of employing the latest technology. The solution is to include the effects of autocorrelation in the analysis.

Another concern when evaluating animal resource selection is assessing the availability of a resource. A common heuristic definition of selection is resource use compared to resource availability. Initial resource selection methods were based on the animal selecting one of several categorical habitats in a study area (Neu, Byers, and Peek, 1974; Byers, Steinhorst, and Krausman, 1984). Availability of a habitat was defined as the area classified as that particular habitat. Christ, Ver Hoef, and Zimmerman (2005) note that the concept of availability is not as well defined when resources are not denoted by categorical habitat designations. The inclusion of contin- uously valued environmental resources as well as the concept of an animal home range and movement confuse the issue of what resources are actually available to an animal (Christ et al., 2005). Using different definitions of availability can lead to different inferences of selection (Porter and Church, 1987; Buskirk and Millspaugh, 2006).

In recognition of the previously mentioned difficulties, as well as other concerns with selection modeling, several alternative models and modifications were proposed (see Section 1.3 of Christ et al., 2005). The discrete choice (DC) model was proposed by McCracken, Manly, and Vander Heyden (1998) to allow availability to change over time, as well as the inclusion of continuously valued resources. Ramsey and Usner (2003) proposed an extension to the DC model that accounts for serial correlation in categorical habitat data. To account for both home range and serial correlation in animal locations in selection inference Christ et al. (2005) and Christ, Ver Hoef, and Zimmerman (2008) proposed a weighted distribution model. Rhodes et al. (2005) proposed a similar, but slightly simpler, model with known home range centers. All of these models illustrate different approaches to dealing with inference from complex telemetry data. Unfortunately, there is no general model for this type of data that researchers can tailor to meet their specific needs.

In this article, we propose a general modeling framework that can be used for analyzing resource selection using animal telemetry data. The model is composed of flexible 
components for both resource selection and movement. All of the models presented will be for the analysis of data from a single individual. Thomas, Johnson, and Griffith (2006) and Ramsey and Usner (2003) use random effects models to make population-level inference from multiple independent animals. These methods can be applied to the individual models we are considering. We, however, do not pursue that aspect in this article.

The article proceeds in the following manner. In Section 2, we develop the proposed framework and associated model notation. Researchers can use the framework to build extremely complex and flexible models but still maintain a cohesive structure that can be interpreted (and critiqued) throughout the ecological literature. Next, Section 3 briefly examines some aspects of parameter estimation. Section 4 illustrates that our proposed model is a generalization of many traditional resource selection and animal movement models. In Section 5, a small simulation experiment is conducted. An example analysis, using the models developed in this article, is presented in Section 6. Finally, we conclude with some comments in Section 7.

\section{Model Development}

\subsection{A Weighted Distribution Approach}

Resource selection functions (RSF) were first introduced by McDonald, Manly, and Raley (1990) as an application of a broader body of statistical literature known as weighted distributions (Patil, 2002). In the resource selection context one can envision a "use" distribution with density $f_{u}(\mathbf{x})$ for a suite of resources $\mathbf{x}$. This is the distribution of resource values that an animal actually uses. The resources are selected by the animal from a distribution of available resources that has density $f_{a}(\mathbf{x})$. The RSF $w(\mathbf{x})$ transforms $f_{a}$ to $f_{u}$ by selectively weighting different resources, i.e.,

$$
f_{u}(\mathbf{x})=K^{-1} w(\mathbf{x}) f_{a}(\mathbf{x})
$$

where $K$ is a normalizing constant. Web Appendix A gives some interesting interpretations of the selection function as distance measures between $f_{u}$ and $f_{a}$.

\subsection{Incorporating Movement and Behavioral Effects}

In order to accommodate animal movement and home range constraints the weighted distribution model can be placed in a spatiotemporal context where the resource, $\mathbf{x}$, corresponds to a spatial location, s, in a study area. Christ et al. (2005, 2007) and Rhodes et al. (2005) present similar models for including home range and movement, both of which are special cases of the following general formulation. By explicitly including movement and home range constraints via modeling, the data can speak to the level and uncertainty of serial correlation and home range scale and seamlessly pass that information to uncertainty in selection parameters.

For the time being we will consider movement only. In a study area $\mathcal{S}$ of area $A$, let a specific location be designated by $\mathbf{s}$ and $\mathbf{x}(\mathbf{s})$ denote a vector of resource covariates that can be measured at $\mathbf{s}$. An animal is located in the study area $T$ times, not necessarily equally spaced. Denote the location of the animal at time $t$ as $\mathbf{s}_{t}$. Further, let $\mathcal{H}_{t-1}=\left\{\mathbf{s}_{1}, \ldots, \mathbf{s}_{t-1}\right\}$ be the history of locations for the animal prior to time $t$. Denote the probability density of locations used by the animal at time $t$ conditional on the history of used locations during the study period as $g_{u}\left(\mathbf{s}_{t} \mid \mathcal{H}_{t-1}\right)$. Now, using the same framework as (1), location use is modeled as a weighted version of the available location density, $g_{a}$, which depends on movement constraint parameters to give

$$
g_{u}\left(\mathbf{s}_{t} \mid \mathcal{H}_{t-1}\right)=K_{t}^{-1} w\left(\mathbf{s}_{t} \mid \mathcal{H}_{t-1}\right) g_{a}\left(\mathbf{s}_{t} \mid \mathcal{H}_{t-1}\right),
$$

where $w\left(\mathbf{s}_{t} \mid \mathcal{H}_{t-1}\right)$ is an $\operatorname{RSF}$ and $K_{t}=\int_{\mathcal{S}} w\left(\mathbf{u} \mid \mathcal{H}_{t-1}\right) \times$ $g_{a}\left(\mathbf{u} \mid \mathcal{H}_{t-1}\right) d \mathbf{u}$. To include a home range effect, one might select a movement model $g_{a}$ that possesses a temporal limiting distribution (see Section 4.4 for example).

Using a second iteration of weighting one can also account for changes in behavior. Suppose the animal exhibits $B$ different behaviors, then a behavioral model can be constructed by additional weighting of $B$ different movement/selection models. This results in the following behavior/movement/selection model,

$$
g_{u}\left(\mathbf{s}_{t} \mid \mathcal{H}_{t-1}\right)=\sum_{b=1}^{B} \vartheta_{t}^{(b)} g_{u}^{(b)}\left(\mathbf{s}_{t} \mid \mathcal{H}_{t-1}\right)
$$

where $\vartheta_{t}^{(b)} \in[0,1], \sum_{b} \vartheta_{t}^{(b)}=1$, and the form of $g_{u}^{(b)}\left(\mathbf{s}_{t} \mid \mathcal{H}_{t-1}\right)$ is given by $(2)$ for each $b=1, \ldots, B$. This formulation allows the researcher to include auxiliary behavioral data, $y_{t} \in$ $\{1, \ldots, B\}$, by letting $\vartheta_{t}^{(b)}=I_{\{b\}}\left(y_{t}\right)$, the indicator function for behavior $b=1, \ldots, B$. Blackwell $(1997,2003)$ proposes a model of this type for animal movement without selection (i.e., $w^{(b)}$ constant in $g_{a}^{(b)}\left(\mathbf{s} \mid \mathcal{H}_{t-1}\right)$ for each $\left.b\right)$.

\section{Estimation and Inference}

We begin our investigation into inference for the general class of proposed selection models with maximum likelihood estimation. Until now we have omitted any parameter notation. For the remainder of the article, however, we allow $w(\mathbf{x})$ to depend on a parameter vector $\boldsymbol{\theta}_{w}$ and $f_{a}$ to depend on a parameter vector $\boldsymbol{\theta}_{a}$. Because the observed data are animal use locations, the resulting likelihood function is

$$
L\left(\boldsymbol{\theta} ; \mathcal{H}_{T}\right)=g_{u}\left(\mathbf{s}_{1}\right) \prod_{t=2}^{T} g_{u}\left(\mathbf{s}_{t} \mid \mathcal{H}_{t-1}\right),
$$

where $\boldsymbol{\theta}=\left(\boldsymbol{\theta}_{w}, \boldsymbol{\theta}_{a}\right)$ and $g_{u}\left(\mathbf{s}_{1}\right)$ is the marginal density function for the first location. If the selection movement model is stationary then the $g_{u}\left(\mathbf{s}_{1}\right)$ would be the temporal limiting distribution.

For Markov models (those that depend on $\mathcal{H}_{t-1}$ only through $\mathbf{s}_{t-1}$ ) Christ et al. (2005) propose using the conditional likelihood obtained by simply removing the $g_{u}\left(\mathbf{s}_{1}\right)$ term in (4). They note that the loss of information in this approach is small if the time series is long. Another approach, which we take in Section 5 , is to use $g_{u}\left(\mathbf{s}_{1}\right) \approx w\left(\mathbf{s}_{1}\right) g_{a}^{\infty}\left(\mathbf{s}_{1}\right)$, where $g_{a}^{\infty}(\mathbf{s})$ is the temporal limiting distribution of the movement model $g_{a}\left(\mathbf{s}_{t} \mid \mathcal{H}_{t-1}\right)$. A small number of simulations have shown this to be a fair approximation.

Estimation methods in resource selection studies often employ an availability sample (locations randomly sampled by the researcher) to approximate the study area. Logistic regression is then used to estimate selection parameters (Manly et al., 2002). There has been considerable debate in the literature recently concerning this approach (see discussions in 
Keating and Cherry, 2004; Johnson et al., 2006; Thomas and Taylor, 2006). Johnson et al. (2006) provide a proof that, under certain simplifying constraints, the maximum likelihood estimator (MLE) derived from a logistic regression likelihood is equivalent to the MLE derived from the true likelihood for animal and researcher selections, provided the researcher draws an availability sample from $g_{a}$. If $g_{a}$ is unknown then it is not possible to justify use of logistic regression with the proof of Johnson et al. (2006) because it depends on drawing a sample from an unknown distribution.

We believe it is more straightforward to consider using the availability sample as a tool for approximating the normalizing constant in (2). This was initially proposed by McCracken et al. (1998) for use with a DC model (see Section 4.2). Their proposed approximation was constructed from a design based estimator of the normalizing constant. We propose the following generalization using importance sampling methods (Givens and Hoeting, 2005, p. 163).

(i) For each animal selected location, $\mathbf{s}_{t}$, independently draw $M$ "available" locations, $\mathbf{u}_{t j}, j=1, \ldots, M$, from $q_{t}\left(\cdot \mid \mathcal{H}_{t-1}\right)$, where $q_{t}\left(\cdot \mid \mathcal{H}_{t-1}\right)$ is a density that is easy to sample from and shares the same support as $g_{u}\left(\cdot \mid \mathcal{H}_{t-1}\right)$.

(ii) Obtain the Monte Carlo MLE (MCMLE) $\hat{\boldsymbol{\theta}}_{m c}$ by maximizing $L_{m c}\left(\boldsymbol{\theta} ; \mathcal{H}_{T}\right)$, which equals (4) with the exception that, for $t=1, \ldots, T, K_{t}$ is replaced with the approximation

$$
\begin{aligned}
\hat{K}_{t}=\frac{1}{M+1}\left\{\sum_{j=1}^{M} \frac{w\left(\mathbf{u}_{t j} \mid \boldsymbol{\theta}\right) g_{a}\left(\mathbf{u}_{t j} \mid \mathcal{H}_{t-1}, \boldsymbol{\theta}\right)}{q_{t}\left(\mathbf{u}_{t j} \mid \mathcal{H}_{t-1}\right)}\right. \\
\left.+\frac{w\left(\mathbf{s}_{t} \mid \boldsymbol{\theta}\right) g_{a}\left(\mathbf{s}_{t} \mid \mathcal{H}_{t-1}, \boldsymbol{\theta}\right)}{q_{t}\left(\mathbf{s}_{t}\right)}\right\} .
\end{aligned}
$$

The strong law of large numbers states that, as $M \rightarrow \infty, \hat{K}_{t} \rightarrow$ $K_{t}$ almost surely (a.s.) for all $t$. If $g_{u}$ is in the exponential family of distributions then Geyer and Thompson (1992) state that $\hat{\boldsymbol{\theta}}_{m c} \stackrel{\text { a.s. }}{\rightarrow} \hat{\boldsymbol{\theta}}$, the MLE based on the exact normalizing constant. In addition, if mild regularity conditions hold then convergence of likelihood gradients and the Hessian matrix are also realized (Geyer and Thompson, 1992). This allows standard error calculation using the Hessian matrix.

It should be noted that one need not actually draw separate samples for each animal location. All of the asymptotic results still hold if one large sample from, say $q(\mathbf{u})$, is drawn regardless of animal locations. Efficiency of approximation will increase, however, with availability samples that track the path of the animal. Essentially, the theory of importance sampling states that the best approximation $q_{t}\left(\cdot \mid \mathcal{H}_{t-1}\right)$ is $g_{u}\left(\cdot \mid \mathcal{H}_{t-1}\right)$, so, availability sample distributions "close" to the actual use distributions will approximate the normalizing constant more efficiently.

\section{Past, Present, and Future}

In this section, we explore different specifications of the general movement model (3) that lead to previously proposed resource selection models. By exploring these links, it enables one to see what assumptions are being made when these models are employed for resource selection studies.

\subsection{Categorical Habitat Model}

Numerous indices have been proposed and compared for describing categorical habitat selection (Manly et al., 2002). Alldredge, Thomas, and McDonald (1998) provide the following common assumptions for studies of categorical habitat selection:

(i) Relocations of an animal are independent over time.

(ii) Every location $\mathbf{s} \in \mathcal{S}$ is available to every animal at every time.

(iii) Each location is uniquely characterized into resource type $h=1, \ldots, H$.

(iv) The selection function, $w$, depends on the location $\mathbf{s}$ only through its habitat class designation.

Under these four assumptions one obtains $g_{a}\left(\mathbf{s}_{t} \mid \mathcal{H}_{t-1}\right)=$ $A^{-1}$, hence,

$$
g_{u}\left(\mathbf{s}_{t} \mid \mathcal{H}_{t-1}\right) \propto w_{h} A_{h} / \sum_{k=1}^{H} w_{k} A_{k} \quad h=1, \ldots, H,
$$

where $A_{h}$ is the area of $\mathcal{S}$ that is characterized by resource type $h$ and $w_{h}$ is the value of the RSF for resource $h$. If the animal is located $T$ times then (4) reduces to

$$
L_{C A T}(w) \propto \prod_{h=1}^{H}\left(\frac{w_{h} A_{h}}{\sum_{k=1}^{H} w_{k} A_{k}}\right)^{C_{h}}
$$

where $C_{1}, \ldots, C_{H}$ are the number of times the animal is located in each resource type.

The main benefit of this model is its simplicity and closed form estimator. Many researchers have noted, however, that the assumptions are often unrealistic. Also, continuously valued resources cannot be considered (i.e., distance to water, elevation, or percent vegetation cover).

\subsection{Discrete Choice Models}

The DC model has been proposed as a generalization of the categorical model (McCracken et al., 1998; Cooper and Millspaugh, 1999; Manly et al., 2002). Here, we illustrate how, under specific assumptions, our general model reduces to a DC model. The assumptions needed are as follows:

(i) The number of locations $\mathbf{s} \in \mathcal{S}$ is of finite size $N$.

(ii) At each time $t$ the animal has a set of locations (choice set), $\mathcal{C}_{t}$, from which to select the next location. All $\mathrm{s} \in$ $\mathcal{C}_{t}$ are equally available. The choice set $\mathcal{C}_{t}$ may depend on $\mathcal{H}_{t-1}$.

If these assumptions are met then $g_{a}\left(\mathbf{s}_{t} \mid \mathcal{H}_{t-1}\right)=$ $n_{t}^{-1} I_{\mathcal{C}_{t}}\left(\mathbf{s}_{t}\right)$, where $n_{t}$ is the size of the choice set and $I_{\mathcal{C}_{t}}\left(\mathbf{s}_{t}\right)$ is an indicator function for the inclusion of $\mathbf{s}_{t}$ in $\mathcal{C}_{t}$. If we now model $w\left(\mathbf{s}_{t} \mid \mathcal{H}_{t-1}\right)=\exp \left\{\mathbf{x}\left(\mathbf{s}_{t}\right)^{\prime} \boldsymbol{\alpha}\right\}$, then the resulting use density is given by

$$
g_{u}\left(\mathbf{s}_{t} \mid \mathcal{H}_{t-1}\right)=\frac{\exp \left\{\mathbf{x}\left(\mathbf{s}_{t}\right)^{\prime} \boldsymbol{\alpha}\right\} I_{\mathcal{C}_{t}}\left(\mathbf{s}_{t}\right)}{\sum_{\mathbf{u} \in \mathcal{S}} \exp \left\{\mathbf{x}(\mathbf{u})^{\prime} \boldsymbol{\alpha}\right\} I_{\mathcal{C}_{t}}(\mathbf{u})} .
$$


Thus, general likelihood (4) reduces to

$$
L_{D C}(\boldsymbol{\alpha})=\prod_{t=1}^{T} \frac{\exp \left\{\mathbf{x}\left(\mathbf{s}_{t}\right)^{\prime} \boldsymbol{\alpha}\right\}}{\sum_{\mathbf{u} \in \mathcal{C}_{t}} \exp \left\{\mathbf{x}(\mathbf{u})^{\prime} \boldsymbol{\alpha}\right\}}
$$

Although locations can be made dependent by allowing $\mathcal{C}_{t}$ to depend on previous locations, the way in which choice sets are constructed, however, must be completely known to the researcher.

\subsection{Ramsey and Usner's Persistence (PER) Model}

Ramsey and Usner (2003) propose a DC model with a PER parameter to model the tendency of animals to stay in the same habitat type between successive relocations. In this section, we illustrate how, under specific assumptions, (3) reduces to the PER model. The assumptions needed are as follows:

(i) At each time the animal selects a new location from $\mathcal{S}$.

(ii) The animal follows two behaviors:

(a) with probability $\vartheta$, select a location within $\mathcal{S}$ where resources are identical to the previous location and

(b) with probability $1-\vartheta$ select a new location following a DC model.

Under these assumptions the movement model is given by

$$
g_{a}\left(\mathbf{s}_{t} \mid \mathcal{H}_{t-1}\right)=\left\{\begin{array}{ll}
I_{\mathcal{R}_{t}}\left(\mathbf{s}_{t}\right) / r_{t} & \text { with prob. } \vartheta \\
I_{\mathcal{C}_{t}}\left(\mathbf{s}_{t}\right) / n_{t} & \text { with prob. } 1-\vartheta
\end{array},\right.
$$

where $\mathcal{R}_{t}$ is the set of all locations in $\mathcal{S}$ such that the habitat classification is the same as $\mathbf{x}\left(\mathbf{s}_{t-1}\right)$ and $r_{t}$ is the size of $\mathcal{R}_{t}$. Now, the selection function is given by

$$
w\left(\mathbf{s}_{t}\right)=\left\{\begin{array}{ll}
1 & \text { with prob. } \vartheta \\
\exp \left\{\mathbf{x}\left(\mathbf{s}_{t}\right)^{\prime} \boldsymbol{\alpha}\right\} & \text { with prob. } 1-\vartheta
\end{array} .\right.
$$

Combining the movement and selection models gives

$$
g_{u}\left(\mathbf{s}_{t} \mid \mathcal{H}_{t-1}\right)=\vartheta \frac{I_{\mathcal{R}_{t}}\left(\mathbf{s}_{t}\right)}{r_{t}}+(1-\vartheta) \frac{\exp \left\{\mathbf{x}\left(\mathbf{s}_{t}\right)^{\prime} \boldsymbol{\alpha}\right\}}{\int_{\mathbf{u} \in \mathcal{S}} \exp \left\{\mathbf{x}(\mathbf{u})^{\prime} \boldsymbol{\alpha}\right\}},
$$

which has the form of the behavior/movement/selection model in (3). Using (4) the PER likelihood is

$$
\begin{aligned}
L_{P E R}(\boldsymbol{\alpha}, \vartheta) \propto & \prod_{t=1}^{T} \vartheta I_{\left\{x_{t-1}\right\}}\left(x_{t}\right)+(1-\vartheta) \\
& \times \frac{\exp \left\{\sum_{h=1}^{H} \alpha_{h} I_{\{h\}}\left(x_{t}\right)+\log \left(A_{h} / A\right)\right\}}{\sum_{h=1}^{H} \exp \left\{\alpha_{h}+\log \left(A_{h} / A\right)\right\}},
\end{aligned}
$$

where $x_{t}=\mathbf{x}\left(\mathbf{s}_{t}\right) \in\{1, \ldots, H\}$ is the sequence of habitat types in which the animal was located. Aside from some small notational differences, this is the model Ramsey and Usner propose.

The PER model generally does not make sense for a point level model. Essentially, an animal would have to remain in exactly the same spot as the previous location with probability $\vartheta$; an unlikely event. In addition, the researcher still needs to completely define a known availability set.

\subsection{Gaussian Process Movement Models}

Here we propose a new model for analyzing resource selection from telemetry data. Using the weighted distribution approach, building a model that incorporates all of the improvements of previous resource selection modeling while eliminating many of the pitfalls is straightforward. First, a bivariate Gaussian process model is used for animal movement. Dunn and Gibson (1977) proposed an Ornstein-Uhlenbeck model (OU), to incorporate home range in animal movement models. Blackwell (1997) improved the OU model by allowing for alternate behaviors in the same manner as Section 2.2 (see Section 6 for example). Here we will focus on the single behavior model (2), but the extension to (3) is straightforward (see Section 6).

The OU movement selection model is defined for observation times $\tau_{1}, \ldots, \tau_{T}$ as follows:

(a) $g_{a}\left(\mathbf{s}_{t} \mid \mathcal{H}_{t-1}\right)=N\left(\boldsymbol{\mu}_{t}, \boldsymbol{\Lambda}_{t}\right)$,

(b) $w\left(\mathbf{s}_{t}\right)=\exp \left\{\mathbf{x}(\mathbf{s})^{\prime} \boldsymbol{\alpha}\right\}$

where $\boldsymbol{\mu}_{t}=\boldsymbol{\mu}+\mathbf{B}_{t}\left(\mathbf{s}_{t-1}-\boldsymbol{\mu}\right), \boldsymbol{\Lambda}_{t}=\boldsymbol{\Lambda}-\mathbf{B}_{t} \boldsymbol{\Lambda} \mathbf{B}_{t}$, and $\mathbf{B}_{t}=\operatorname{Diag}\left(\exp \left\{-\left(\tau_{t}-\tau_{t-1}\right) / \phi\right\}\right)$. Blackwell (2003) notes that a single autocovariance parameter $\phi$ is usually sufficient.

One can interpret $\boldsymbol{\mu}$ and $\boldsymbol{\Lambda}$ as defining the center and spread, respectively, of an elliptical home range. Technically, however, $\boldsymbol{\mu}$ represents a central attraction point and $\boldsymbol{\Lambda}$ is a positive definite matrix that controls the strength of attraction from various locations in the study area. The limiting distribution, $g_{a}^{\infty}(\cdot)$, for the OU process is $N(\boldsymbol{\mu}, \boldsymbol{\Lambda})$. The range of correlation in locations can be inferred from $\phi$ by noting $\operatorname{Corr}\left(\mathbf{s}_{\tau}, \mathbf{s}_{\tau+3 \phi}\right) \approx 0$. Therefore, at a time separation of $3 \phi$, locations can be considered independent. Christ et al. (2005, 2007) consider a model similar to the OU model by using a vector autoregressive (VAR) movement model. In fact, for uniform observation times, the OU model can be reparameterized to VAR form. One benefit of the OU form is that it automatically handles nonuniform observation times.

There are additional benefits of the OU movement selection model. First, like the PER model, autocorrelation is included in the model. Unlike the PER model, however, the autocorrelation is built in at the point level from physical principles of motion. Second, like the DC model all environmental variables, categorical and continuous, as well as spatial resolutions, can be modeled without loss of interpretability. Unlike the DC model, however, choice sets do not need to be defined by the user. The data speak to the availability of resources via the estimated $\boldsymbol{\Lambda}$ and $\phi$.

Another Gaussian process that would be useful for animals with no home range effects is a standard Brownian motion (BM) model. The BM model is very similar to the $\mathrm{OU}$ model. Essentially, the same model is used with $\boldsymbol{\mu}_{t}=\mathbf{s}_{t-1}$ and $\boldsymbol{\Lambda}_{t}=\boldsymbol{\Lambda}\left(\tau_{t}-\tau_{t-1}\right)$. Usually, one also uses the constraint that $\boldsymbol{\Lambda} \propto \mathbf{I}$, but this is not necessary. The BM movement model does not have a stationary distribution so the likelihood must condition on $\mathbf{s}_{1}$. 
Table 1

Bias and RMSE properties of selection coefficient maximum likelihood estimators for the simulated data sets

\begin{tabular}{lllcrr}
\hline \hline & & \multicolumn{5}{c}{ Independence range } \\
\cline { 3 - 6 } Estimator property & Model & 20 & 10 & 5 & 1 \\
\hline Relative bias $\left(\alpha_{1}\right)$ & OU & 0.01 & 0.01 & -0.01 & 0.03 \\
& DC & -0.2 & -0.28 & -0.36 & -0.34 \\
& PER & -0.19 & -0.28 & -0.36 & -0.34 \\
Relative bias $\left(\alpha_{2}\right)$ & OU & -0.07 & 0.01 & -0.09 & 0.02 \\
& DC & -0.95 & -0.99 & -1.23 & -1.17 \\
RMSE $\left(\alpha_{1}\right)$ & PER & -0.95 & -0.99 & -1.23 & -1.17 \\
& OU & 0.29 & 0.26 & 0.24 & 0.3 \\
RMSE $\left(\alpha_{2}\right)$ & DC & 0.46 & 0.5 & 0.59 & 0.59 \\
& PER & 0.44 & 0.5 & 0.59 & 0.59 \\
& OU & 0.36 & 0.31 & 0.36 & 0.32 \\
& DC & 0.87 & 0.82 & 0.99 & 0.94 \\
& PER & 0.85 & 0.82 & 1.00 & 0.93 \\
\hline
\end{tabular}

Table 2

Power and $95 \%$ confidence interval coverage of selection coefficient maximum likelihood estimators for simulated data sets

\begin{tabular}{lllccc}
\hline \hline & & \multicolumn{4}{c}{ Independence range } \\
\cline { 3 - 6 } Estimator property & Model & 20 & 10 & 5 & 1 \\
\hline Power $\left(\alpha_{1}\right)$ & OU & 1.00 & 1.00 & 1.00 & 1.00 \\
& DC & 0.99 & 0.97 & 0.98 & 0.97 \\
\multirow{2}{*}{ Power $\left(\alpha_{2}\right)$} & PER & 0.98 & 0.97 & 0.98 & 0.97 \\
& OU & 0.51 & 0.65 & 0.56 & 0.63 \\
& DC & 0.07 & 0.03 & 0.01 & 0.01 \\
$95 \%$ CI coverage $\left(\alpha_{1}\right)$ & PER & 0.06 & 0.00 & 0.01 & 0.01 \\
& OU & 0.96 & 0.94 & 0.99 & 0.91 \\
& DC & 0.66 & 0.64 & 0.37 & 0.43 \\
$95 \%$ CI coverage $\left(\alpha_{2}\right)$ & PER & 0.78 & 0.68 & 0.4 & 0.46 \\
& OU & 0.97 & 0.98 & 0.92 & 0.95 \\
& DC & 0.43 & 0.38 & 0.19 & 0.19 \\
& PER & 0.54 & 0.44 & 0.23 & 0.19 \\
\hline
\end{tabular}

The general likelihood for a Gaussian process model is,

$$
\begin{aligned}
& L_{G P}\left(\boldsymbol{\alpha}, \boldsymbol{\theta}_{G P}\right) \\
& =\prod_{t=1}^{T} \frac{\exp \left\{\mathbf{x}\left(\mathbf{s}_{t}\right)^{\prime} \boldsymbol{\alpha}+\left(\mathbf{s}_{t}-\boldsymbol{\mu}_{t}\right)^{\prime} \boldsymbol{\Lambda}_{t}^{-1}\left(\mathbf{s}_{t}-\boldsymbol{\mu}\right) / 2\right\}}{\int_{\mathcal{S}} \exp \left\{\mathbf{x}(\mathbf{u})^{\prime} \boldsymbol{\alpha}+\left(\mathbf{u}-\boldsymbol{\mu}_{t}\right)^{\prime} \boldsymbol{\Lambda}_{t}^{-1}(\mathbf{u}-\boldsymbol{\mu}) / 2\right\} d \mathbf{u}},
\end{aligned}
$$

where $\boldsymbol{\theta}_{G P}$ are the movement parameters used to model $\boldsymbol{\mu}_{t}$ and $\boldsymbol{\Lambda}_{t}$. Note that the Gaussian process models have the form of a DC model with anisotropic distance to the last location as a covariate. If Euclidean distance were used as a covariate (see Example 8.2 in Manly et al. [2002]) it would be equivalent to using a bivariate Laplace distribution centered on $\mathbf{s}_{t-1}$ for the movement model.

\section{A Simulation Experiment}

In order to demonstrate the benefits of using the framework to construct more realistic models for selection from teleme- try data we provide the results of a simulation experiment. The simulation experiment provides a solid base for judging whether a more complex model, such as the OU model, allows a researcher to make better inference concerning resource selection.

\subsection{Details of the Experiment}

In the experiment we compared three models: DC, PER, and OU. The same RSF, $w(s)=\exp \left\{\mathbf{x}(\mathbf{s})^{\prime} \boldsymbol{\alpha}\right\}$, was used throughout all simulations. Animal paths were simulated from a discretized version of the OU model on a $100 \times 100$ grid of locations. The MLE for each path was calculated from (4) for each model. For the OU model we used $g_{u}\left(\mathbf{s}_{1}\right) \propto w\left(\mathbf{s}_{1}\right) g_{a}^{\infty}\left(\mathbf{s}_{1}\right)$. Models were compared using relative bias ([simulation average true value]/true value), root mean square error (RMSE), power to detect positive selection coefficients (one-tailed test, $5 \%$ significance level), and $95 \%$ CI coverage. Because the data are simulated from the OU model it was fully expected that the OU model would perform the best. What was unknown, however, was the degree to which it would outperform the others.

A study area with three categorical habitat levels on which paths were simulated was constructed. The environment included equal amounts of the three habitat types. Next, 100 paths of $T=100$ observations were simulated under four levels of autocorrelation, $\phi=20 / 3,10 / 3,5 / 3$, and $1 / 3$. These correspond to independence at roughly 20, 10, 5, and 1 time units. For the home range parameters we set $\boldsymbol{\mu}=(50,50)$, $\boldsymbol{\Lambda}=200 \mathbf{I}$. Finally, and most importantly, the selection parameters were set at $\boldsymbol{\alpha}=(0,1.5,0.75)$. The coefficient $\alpha_{1}$ was held fixed at zero for identifiability (implying the remaining coefficients represent log relative selection ratios). See Web Figure 1 for example.

\subsection{Results of the Experiment}

The results of the selection parameter comparisons are presented in Tables 1 and 2. As expected, the OU model performed better than the DC or PER models. The magnitude of the DC and PER model shortcomings was, however, unexpected. In terms of relative bias, RSME, power, and CI coverage, the OU model gave significant improvements in selection parameter inference.

The OU model estimates are approximately unbiased for each dependence range (Table 1). RMSE held nearly constant over each of the dependence ranges $(0.24-0.36)$. Table 2 shows that for the largest selection parameter $\left(\alpha_{2}\right)$ power to detect $\alpha_{2}>0$ was uniformly 1.0. The power to detect $\alpha_{3}>0$ ranged from 0.51 to 0.65 . CI coverage ranged from $92 \%$ to $99 \%$ for both coefficients. Web Table 1 contains the results for the OU movement parameters.

The DC and PER models were nearly identical with respect to bias. They both had uniformly negative bias that is positively related to the dependence range. The bias magnitude increases as the observations become independent. Because of this bias, the same phenomenon occurred with RMSE (Table 1 ) and CI coverage rates (Table 2). Power to detect $\alpha_{2}>$ 0 was nearly $100 \%$ for both models at all dependence levels (Table 2). For $\alpha_{3}$, however, power was almost 0\%. Model misspecification and resulting negative bias contributed to power below $5 \%$. CI coverage ranged from approximately $40 \%$ to 


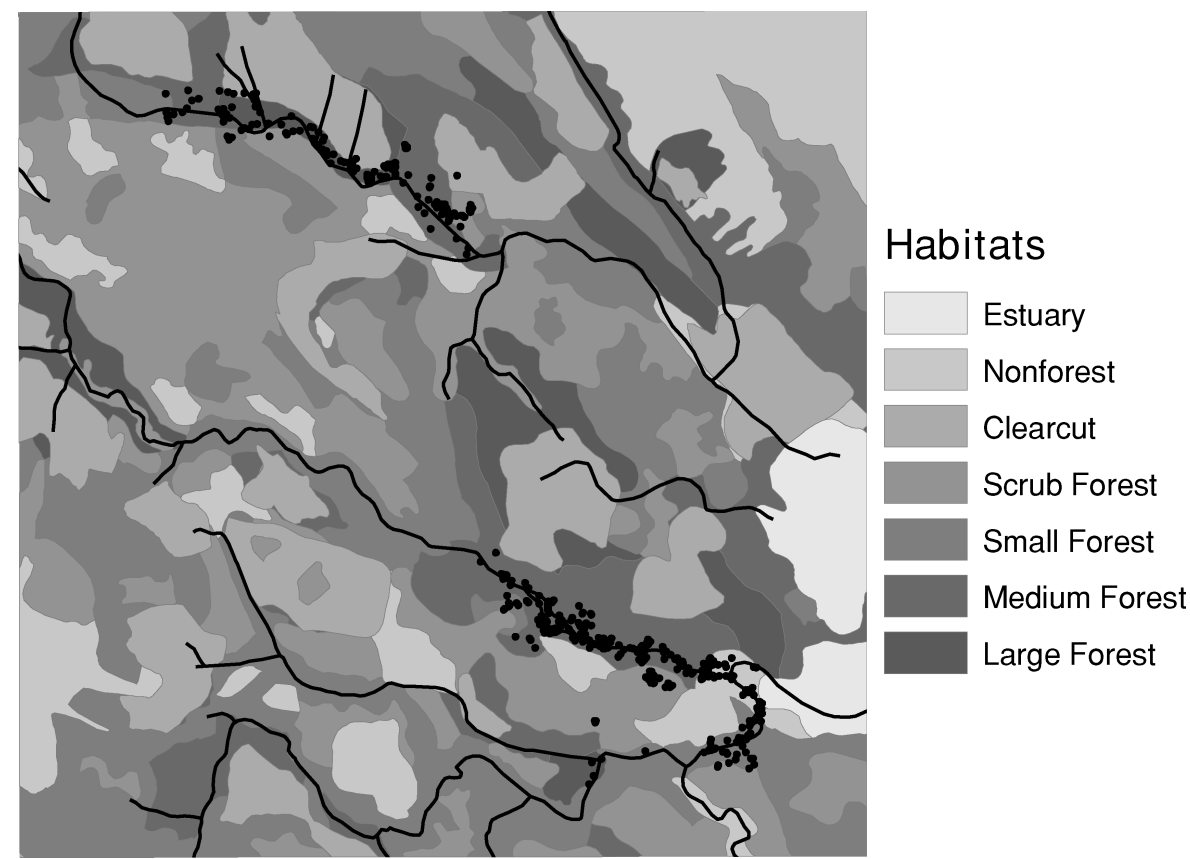

Figure 1. Bear locations for the example data set examined in Section 6 .

$80 \%$ for $\alpha_{2}$ and $20 \%$ to $50 \%$ for $\alpha_{3}$ with coverage higher for the PER model as expected.

\section{Brown Bear Example}

Here we present analysis of selection for a single male brown bear (Ursus arctos) in southeast Alaska. We compared a variety of models that belong to the general class defined by (3). Data were collected, on average, every 1.72 hours, with a range of $0.46-22.01$ hours using a GPS collar. The animal was located on $T=457$ occasions (Figure 1 ).

We are interested in two resources for which selection could be inferred. First, a categorical habitat class is available via a 50-m-resolution GIS layer. The classes included: estuary, nonforest, clearcut, scrub forest, small forest, medium forest, and large forest. Second, perpendicular distance from the nearest stream was also calculated on the centroids of the $50-\mathrm{m}$ grid. In order to make the analysis computationally tractable, the $50-\mathrm{m}$ grid was aggregated to a $256 \times 256$ grid and bear locations were snapped to the centroid of the grid. The cell centroids are then modeled as the only available locations in the same manner as the simulation experiment. Habitat layer aggregation was accomplished by selecting the majority habitat type within cells which were combined. For the stream distance variable, the mean distance was used for aggregation.

In the analysis of the bear data five different selection models from the general class were fit. First, the data were naively modeled with a DC model, which assumes the entire study area was available for each use location. We used this as a base which states that there are no home range or serial correlation effects. Secondly, to include time correlation effects only, we utilized a BM movement model. Finally, to include time and home range effects, an OU movement model was employed. Looking at Figure 1, however, one can see that there is a possible behavioral shift in which the bear moves to another foraging area in the northwest section of the study area. Therefore, three OU models were fit. The first is as described in Section 4.4, a single set of home range parameters (OU1). In the second model, OU2, a known behavior indicator $y_{t} \in\{1$, $2\}$ was employed to model two separate circular home ranges (i.e., $\boldsymbol{\Lambda} \propto \mathbf{I}$ ). Finally, in model OU3, two elliptical home range distributions were modeled using the $y_{t}$ indicator.

Each model was fit to the data using maximum likelihood estimation, where the likelihood function in (4) was used. In order to broadly compare models Akaike information criterion (AIC), and subsequently the Akaike weight, was calculated for each model (Burnham and Anderson, 1998). The bimodal-elliptical home range model, OU3, was clearly the optimal AIC model even with its 18 parameters (Web Table 2). The OU3 model collected $99 \%$ of the Akaike weight for the group of models. It was distantly followed by the circular home range model (OU2; 14 parameters) with $1 \%$ of the weight. The remaining models possessed zero weight of evidence. The DC model was clearly inadequate, implying the existence of strong serial correlation and home range effects.

The parameter estimates along with $p$-values for each fitted model are given in Table 3. One can notice some marked difference in parameter estimates and significance levels between models. For the most part, the naive DC model is overly optimistic in its assessment of significance for most of the selection parameters. When comparing the four movement models there is little disagreement between the selection coefficients in direction and magnitude. There is a general rise in $p$-values for the selection coefficients as one proceeds from low to high model complexity, suggesting that the movement portion of the model gives a better explanation of the data than some of the resource covariates. 
Table 3

Bear data resource selection function parameter estimates. Values in parentheses are p-values for a $Z$ test of difference from zero.

\begin{tabular}{|c|c|c|c|c|c|c|}
\hline \multirow[b]{2}{*}{ Resource } & \multicolumn{6}{|c|}{ Model } \\
\hline & $\mathrm{DC}$ & $\mathrm{BM}$ & OU1 $1^{\mathrm{a}}$ & $\mathrm{OU} 2^{\mathrm{b}}$ & $\mathrm{OU}^{\mathrm{c}}$ & OU3 (MCMLE) \\
\hline \multicolumn{7}{|l|}{ Habitat } \\
\hline Estuary & $-2.34(0.000)$ & $-9.61(0.137)$ & $-8.53(0.641)$ & $-9.10(0.769)$ & $-8.49(0.714)$ & $-9.81(0.675)$ \\
\hline Nonforest & $-0.18(0.339)$ & $-1.08(0.000)$ & $-1.02(0.001)$ & $-0.70(0.026)$ & $-0.69(0.030)$ & $-1.13(0.001)$ \\
\hline Clearcut & $-2.34(0.000)$ & $-0.93(0.004)$ & $-0.92(0.005)$ & $-0.99(0.0044)$ & $-0.91(0.009)$ & $-1.76(0.000)$ \\
\hline Scrub forest & $-0.68(0.000)$ & $-0.58(0.021)$ & $-0.48(0.075)$ & $-0.10(0.7196)$ & $-0.09(0.742)$ & $-0.69(0.021)$ \\
\hline Small forest & $-1.26(0.000)$ & $-0.45(0.131)$ & $-0.36(0.256)$ & $0.07(0.827)$ & $0.08(0.814)$ & $-0.11(0.737)$ \\
\hline Medium forest & $0.40(0.004)$ & $-0.65(0.007)$ & $-0.67(0.006)$ & $-0.43(0.090)$ & $-0.41(0.115)$ & $-0.65(0.013)$ \\
\hline Large forest & $0.00(\mathrm{NA})$ & $0.00(\mathrm{NA})$ & 0.00 (NA) & $0.00(\mathrm{NA})$ & 0.00 (NA) & $0.00(\mathrm{NA})$ \\
\hline Stream distance & $-3.50(0.000)$ & $-1.94(0.000)$ & $-1.88(0.000)$ & $-2.26(0.000)$ & $-2.11(0.000)$ & $-2.39(0.000)$ \\
\hline
\end{tabular}

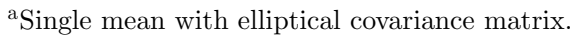

${ }^{\mathrm{b}}$ Two means with circular covariance matrix.

${ }^{\mathrm{c}}$ Two means with elliptical covariance matrix.

${ }^{\mathrm{d}} \mathrm{MC}$ MLE with $M=2000$.

The DC model ignores time and home range effects and presents standard error estimates, hence significance values, which do not reflect realistic parameter uncertainty. The negative nonforest coefficient becomes more significant when time and home range effects are included. This is due to the fact that over the study area as a whole there is not as much nonforest habitat as there is available to the bear when a time dependent availability window is used.

Focusing on the optimal AIC model, OU3, more closely, we can make some biological inference concerning the selection function of the bear. Relative to large forest habitat, there is no significant selection of the other forest classes. There is significantly negative selection of clearcut and nonforest habitat. There is a significant, strongly negative selection of habitat located far from streams. This is expected due to the large dependence of brown bears on salmon in streams. The fact that a bimodal home range was observed implies that there is evidence of multiple spatial scales of selection. The bear seems to first select a larger general "foraging" home range in which small scale selections of habitat are made while it forages. Figure 2 illustrates selection and use distributions of the bear. The strong selection of river corridors is strongly present in Figure 2d.

In order to examine the effect of using an availability sample as an approximation to the exact MLE a sample of $M=$ 2000 locations on the grid was chosen with proposal $q_{t}(\mathbf{u})=$ $A^{-1}$. The MC MLE was calculated using (5). The MCMLE selection coefficients are presented in Table 3 along with the OU3 MLE. For the most part the results are qualitatively the same as the exact MLE. There are, however, some differences. Both scrub and medium forest coefficients have become more negative and significant. Nonforest and clearcut coefficients have also become slightly more negative, but, these were already significantly different from zero, so qualitatively remain unchanged.

The MLE for the movement portion of the OU3 model can also provide some interesting information (Web Table 3 ). The MLE of the autocorrelation parameter $\hat{\phi}=45.89$ which implies that observations $3 \times(45.89 \pm 9.41) \approx 138 \pm 28$ hours apart are roughly independent. MCMLE estimates were very similar to the exact MLE. Area within the $75 \%$ ellipses, $A^{(b)}$ defined by each $\boldsymbol{\mu}^{(b)}$ and $\boldsymbol{\Lambda}^{(b)}$ were also calculated (Web Appendix B). For the bear data, area and $95 \%$ confidence intervals are $\hat{A}^{(1)}=4017.27 \pm 68.42$ and $\hat{A}^{(2)}=1921.56 \pm 70.34$. The first occupied home range was significantly larger than the second $(Z=3.35, p=0.0004)$. Perhaps there was some missing factor leading to a smaller home range and availability window for movement once the bear switched primary location. This could be related to terrain differences or perhaps another bear.

\section{Discussion}

The proposed general framework and associated model take a large step in unifying the methodologically diverse field of animal resource selection. With the proposed model building framework researchers can build models tailored to their particular species of interest, yet the model framework contains common aspects such that comparisons between different studies can be made.

The example analysis presented herein was concerned with data from a single animal. Even with that simplification, obtaining parameter estimates was computationally expensive. Estimating parameters for large (or fine resolution) study areas or large numbers of animals may become problematic. MCMC procedures for multiple animal random effects models such as Thomas et al. (2006) are difficult to implement with computationally expensive likelihoods.

In addition to estimating population level selection parameters with multiple animals Bayesian inference could be used to account for data uncertainty or location prediction. A hierarchical model could be envisioned in which (3) models the true locations of an animal and the observed locations are equal to the true location plus some random error. This approach could also be used with habitat measurement error as well. Predictive distributions are hard to obtain (due to $K_{t}$ ) with the general model. Within an MCMC framework, however, they are relatively easy to obtain. Christ et al. (2005) have used posterior location predictions derived from MCMC simulation to estimate a spatial use distribution that accounts for home range effects as well as selection. This approach could be applied to these models in general. 
( a )
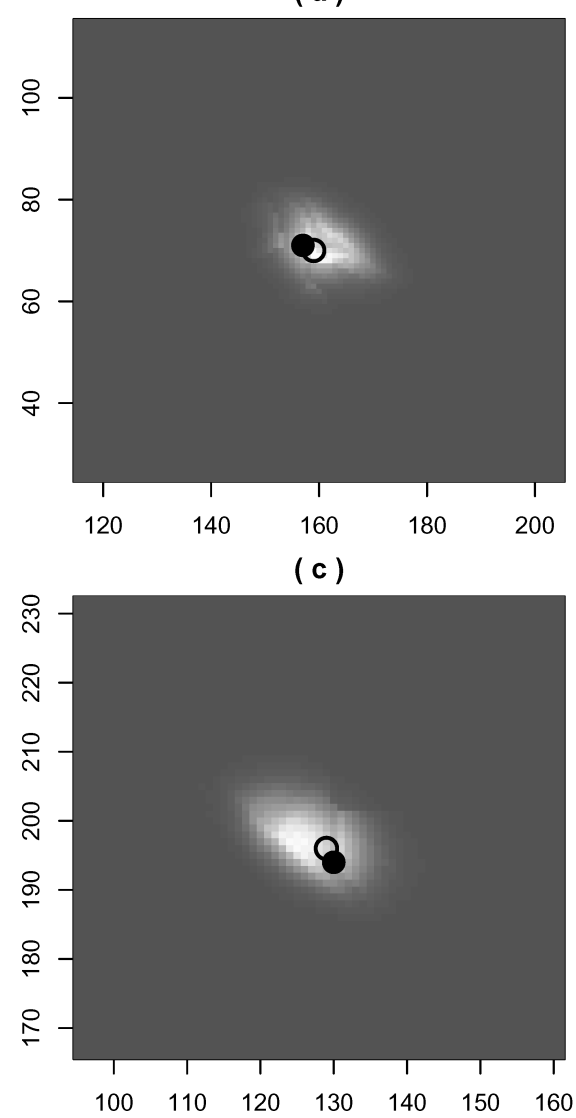

(b)
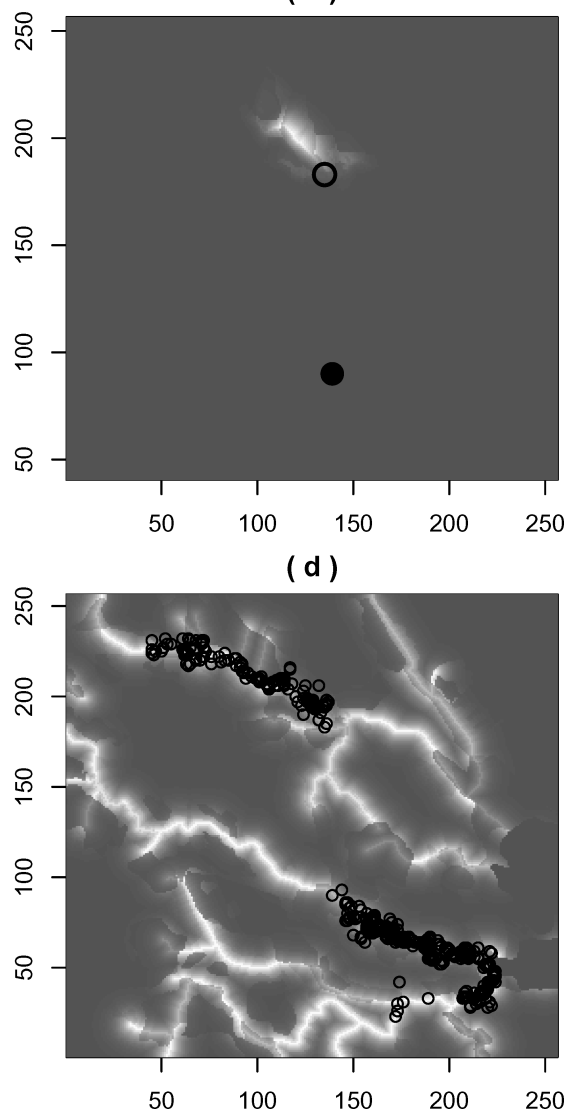

Figure 2. Use and selection for fitted OU3 model in Section 6. Figures (a)-(b) are the estimated use distributions for $t=100,319$, and 440, respectively. The solid dot is the previous location and the open circle is the realized location. Figure (b) illustrates the transition move between primary foraging areas. Figure (d) is the map of $w(\cdot)$ normalized to 1.

The general framework presents a cohesive way to view and develop models for telemetry data now and in the future. Telemetry technology will only improve in the future. Thus the field needs general methodology that has room to expand as the answerable questions become more complex. The proposed framework possesses this type of "room to grow."

\section{Supplementary Material}

Web Tables, Figures, and Appendices A and B, referenced in Sections 2.1, 5.1, and 6, respectively, $\mathrm{R}$ code for implementing the OU model, and the bear data are available under the Paper Information link at the Biometrics website http://www.biometrics.tibs.org.

\section{ACKNOWLEDGEMENTS}

The authors would like to thank J. London, J. Durban, G. Duker, and three anonymous reviewers for comments that improved the article. Also, R. Flynn from ADF\&G graciously provided the bear for the example analysis.

\section{REFERENCES}

Alldredge, J. R., Thomas, D. L., and McDonald, L. L. (1998). Survey and comparison of methods for study of resource selection. Journal of Agricultural, Biological, and Environmental Statistics 3, 237-253.

Blackwell, P. G. (1997). Random diffusion models for animal movement. Ecological Modelling 100, 87-102.

Blackwell, P. G. (2003). Bayesian inference for Markov processes with diffusion and discrete components. Biometrika 90, 613-627.

Burnham, K. and Anderson, D. (1998). Model Selection and Inference. New York: Springer.

Buskirk, S. W. and Millspaugh, J. J. (2006). Defining availability and selecting a currency of use: Key steps in modeling resource selection. Journal of Wildlife Management 70, 358-366.

Byers, C., Steinhorst, R., and Krausman, P. (1984). Clarification of a technique for the analysis of utilizationavailability data. Journal of Wildlife Management 48, 1050-1053.

Christ, A., Ver Hoef, J. M., and Zimmerman, D. (2005). An animal movement model incorporating resource selection and home range. In 2004 Proceedings of the American Statistical Association, Section on Statistics and the Environment [CDROM], Alexandria, VA: American Statistical Association.

Christ, A., Ver Hoef, J. M., and Zimmerman, D. (2008). An animal movement model incorporating home range and 
habitat selection. Environmental and Ecological Statistics, in press.

Cooper, A. B. and Millspaugh, J. J. (1999). The application of discrete choice models to wildlife resource selection studies. Ecology 80, 566-575.

Dunn, J. and Gibson, P. (1977). Analysis of radio-telemetry data in studies of home range. Biometrics 33, 85-101.

Geyer, C. J. and Thompson, E. A. (1992). Constrained Monte Carlo maximum likelihood for dependent data (with discussion). Journal of the Royal Statistical Society, Series $B$ 54, 657-699.

Givens, G. and Hoeting, J. (2005). Computational Statistics. Hoboken, NJ: John Wiley \& Sons, Inc.

Johnson, C., Nielsen, S., Merrill, E., McDonald, T., and Boyce, M. (2006). Resource selection functions based on use-availability data: Theoretical motivation and evaluation methods. Journal of Wildlife Management 70, 347357.

Keating, K. A. and Cherry, S. (2004). Use and interpretation of logistic regression in habitat-selection studies. Journal of Wildlife Management 68, 774-789.

Manly, B., McDonald, L., Thomas, D., McDonald, T., and Erikson, W. (2002). Resource Selection by Animals, 2nd edition. Norwell, MA: Kluwer Academic Publishers.

McCracken, M. L., Manly, B., and Vander Heyden, M. (1998). The use of discrete-choice models for evaluating resource selection. Journal of Agricultural, Biological and Environmental Statistics 3, 268-279.

McDonald, L. L., Manly, B. F. J., and Raley, C. M. (1990). Analyzing foraging and habitat use through selection functions. Studies in Avian Biology 13, 325-331.
Neu, C., Byers, C., and Peek, J. (1974). A technique for analysis of utilization-availability data. Journal of Wildlife Management 38, 541-545.

Patil, G. (2002). Weighted distributions. In Encyclopedia of Environmetrics, A. H. El-Shaarawi and W. Piegorsch (eds). volume 4, 2369-2377. Chichester, UK: John Wiley \& Sons, Ltd.

Porter, W. and Church, K. (1987). Effects of environmental pattern on habitat preference analysis. Journal of Wildlife Management 51, 681-685.

Ramsey, F. and Usner, D. (2003). Persistance and heterogeneity in habitat selection studies using radio telemetry. Biometrics 59, 332-340.

Rhodes, J., McAlpine, C., Lunney, D., and Possingham, H. (2005). A spatially explicit habitat selection model incorporating home range behavior. Ecology 86, 11991205.

Swihart, R. and Slade, N. (1985). Testing for independence in observations of animal movements. Ecology 66, 11761184 .

Thomas, D. L. and Taylor, E. J. (2006). Study designs and tests for comparing resource use and availability II. Journal of Wildlife Management 70, 324-336.

Thomas, D. L., Johnson, D. S., and Griffith, B. (2006). A Bayesian discrete choice model for resource selection with random effects. Journal of Wildlife Management $\mathbf{7 0}$, 404-412.

Received March 2007. Revised July 2007. Accepted September 2007. 